\title{
SciReader enables reading of medical content with instantaneous definitions
}

\author{
Patrick R Gradie ${ }^{1}$, Megan Litster ${ }^{1}$, Rinu Thomas², Jay Vyas², Martin R Schiller ${ }^{\text {* }}$
}

\begin{abstract}
Background: A major problem patients encounter when reading about health related issues is document interpretation, which limits reading comprehension and therefore negatively impacts health care. Currently, searching for medical definitions from an external source is time consuming, distracting, and negatively impacts reading comprehension and memory of the material.

Methods: SciReader was built as a Java application with a Flex-based front-end client. The dictionary used by SciReader was built by consolidating data from several sources and generating new definitions with a standardized syntax. The application was evaluated by measuring the percentage of words defined in different documents. A survey was used to test the perceived effect of SciReader on reading time and comprehension.

Results: We present SciReader, a web-application that simplifies document interpretation by allowing users to instantaneously view medical, English, and scientific definitions as they read any document. This tool reveals the definitions of any selected word in a small frame at the top of the application. SciReader relies on a dictionary of 750,000 unique Biomedical and English word definitions. Evaluation of the application shows that it maps 98\% of words in several different types of documents and that most users tested in a survey indicate that the application decreases reading time and increases comprehension.
\end{abstract}

Conclusions: SciReader is a web application useful for reading medical and scientific documents. The program makes jargon-laden content more accessible to patients, educators, health care professionals, and the general public.

\section{Background}

While $99 \%$ of people in the United States are considered literate, current estimates indicate that only $17 \%-28 \%$ have a basic science literacy and only about 150 million people are what doctors consider medically literate [1-4]. Low scientific and medical literacy renders medical documentation difficult to read and impacts the health care system. Studies link low medical literacy to poor health status, lower self-reporting of medical conditions, lower compliance with doctor's directions, increased rates of hospitalization, and increased health care costs [1]. Medical literacy is partially hindered by the large medical vocabulary, which far exceeds the knowledge boundaries of most people.

\footnotetext{
* Correspondence: martin.schiller@unlv.edu

'School of Life Sciences, University of Nevada, Las Vegas, 4505 Maryland Pkwy., Las Vegas, NV 89154-4004 USA

Full list of author information is available at the end of the article
}

Major initiatives in the United States have yielded a modest increase in literacy by about $15 \%$ since the $1980 \mathrm{~s}$ [1-4]. However, the average American is still not considered scientifically literate. There are now several types of tools that facilitate literacy. Web browsers provide access to millions of documents by anyone with internet access and digital document readers focus on user-friendly presentation of many types of documents. One remaining limitation is the problem of document interpretation. This is true especially in health care where the highly technical terminology often obscures comprehension, and limits understanding to all but a small group of experts.

Readers tend to invoke three general strategies while reading a jargon-rich medical or scientific document. First, the reader may opt to ignore the unknown word altogether. Although this may decrease reading time, it by no means aids in understanding. The second strategy is to infer the meaning of the unknown word from the surrounding text, which is an inexact and error-prone
C Biomed Central 
approach. Finally, a person may decide to consult an outside source such as a dictionary. This strategy tends to be time consuming and can negatively impact reading comprehension and memory of the material [5].

A literary tool that simplifies interpretation would make jargon-laden content more accessible to patients, educators, health care professionals, as well as the general public. To address this problem, we have built SciReader, a open access web-application that allows users to instantaneously view English, medical, and scientific word definitions as they read any document. This tool reveals the definitions of any selected word in small frame at the top of the application.

\section{Implementation}

\section{Application and Database Design}

The SciReader web server was coded in Java with a Flexbased front end client, which requires the commonly used Flash plug-in. Our initial implementation of SciReader relies on a dictionary of $\sim 750,000$ unique Medical, Biological, and English word definitions. The vocabulary was derived by consolidating data from several sources including WordNet [6], Open Biomedical Ontologies [7], NCI thesaurus [8], Medical Subject Headings [9], and the Gene Ontology [10]. Additional word definitions for numerous protein and genes in RefSeq were generated in a standardized syntax using functions from the Gene Ontology $[10,11]$. These vocabularies were implemented in a MySQL database. The number of word definitions in these sources is shown in Figure 1. SciReader can be readily extended to include scientific vocabularies from any other field.

\section{Word Search Algorithm}

When a user clicks a word in the "Reading Area" the following occurs:

1. Both the clicked word and the sentence the word belongs to are sent to the server.

2. The server then creates a list of possible word phrases by performing a database search for the following words:

a. The selected word.

b. Words that end with the selected word.

c. Words that begin with the selected word.

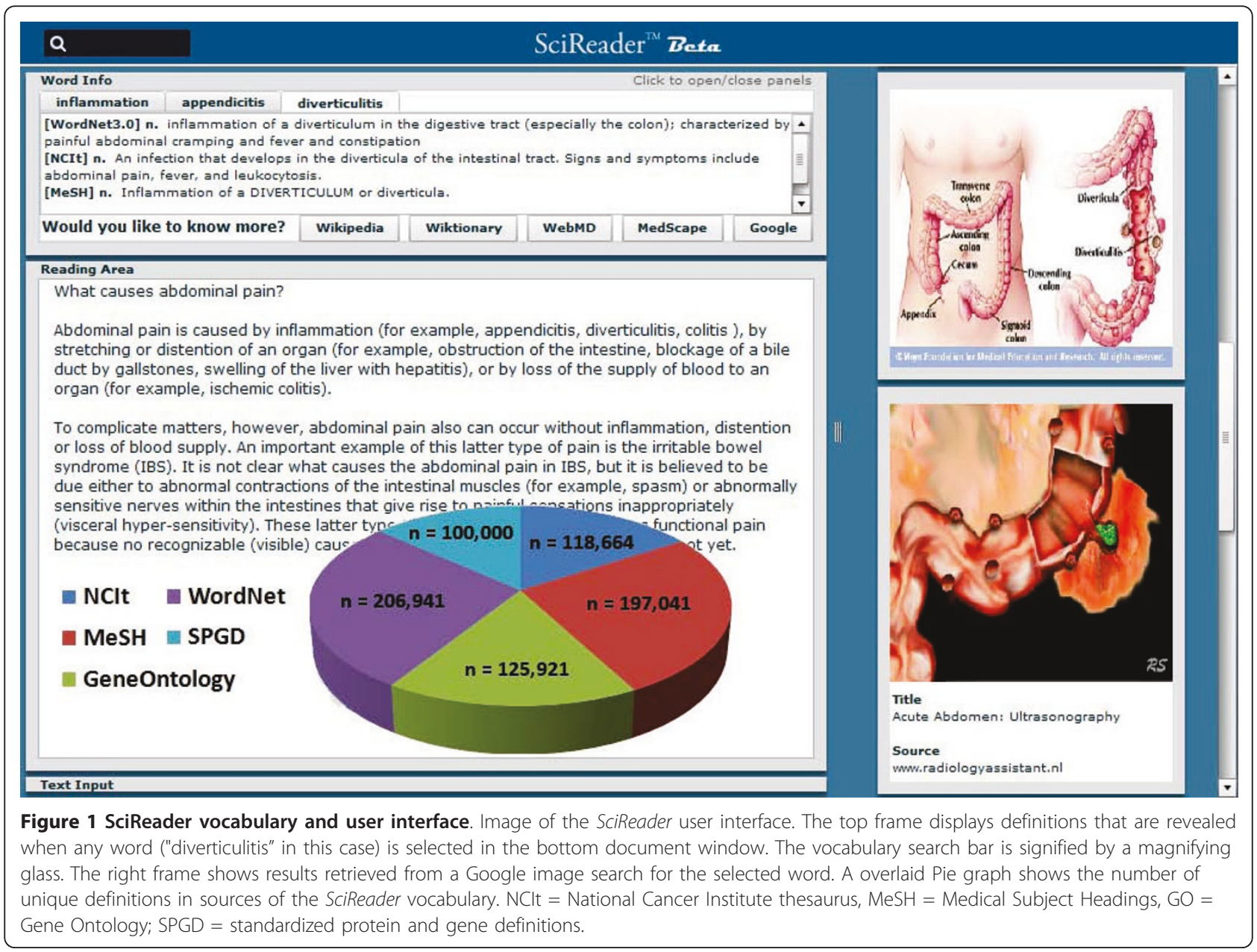


"In order to cull the results returned, when possible, the server will use both the selected word and a word to the right and/or left.

3. Once this set of words is found the longest word phrase length is determined by selecting the longest word phrase length of all the returned word phrases (the word phrase length is returned with the database search for each word phrase).

4. Using the longest word length, a set of word phrases is generated from the sentence by creating all possible word phrases that are at most the length of the longest word phrase length returned from \#3.

5 . Each word generated from \#4 is then matched against the possible word list generated from \#2 and the definitions for each matching word are sent back to the client user.

6. The definitions for each word phrase found in the sentence are shown to the user.

\section{Client Trial}

In order to gauge the effectiveness and usability of SciReader, 105 students in a introductory college biology class (Biology 100 at UNLV) were provided access to the SciReader application and asked to answer a couple of survey questions. The subjects were provided access for $\sim 1$ month to seven chapters in their biology textbook in the SciReader application. Two different survey questions were related to reading comprehension and reading time. Students were asked to respond to the following statements: "I think that using SciReader while reading my science textbook decreased the time it took for me to read." and "I think that using SciReader improved my understanding of the material I read.". Students selected responses from a 5-point Likert scale with 1 = strongly agree, 2 = agree, 3 = neutral, $4=$ disagree, 5 = strongly disagree. An average score was calculated and used as a metric to measure the perceived effectiveness of SciReader. It is important to note that a lower score correspond to a readers agreement with the statement. The survey protocol was approved by the UNLV Social/Behavioural Institutional Review Board (IRB protocol number: 1007-3529M).

\section{Results}

\section{Application Design}

The view of the SciReader user interface shown in Figure 1 contains a small dynamic window frame that displays multiple definitions and a window showing the uploaded text. The application accepts text input from a third window that disappears after text is loaded. Definitions are displayed when any word is selected with a mouse click.

SciReader has a number of basic features that facilitate ease-of-use. In addition to single word definitions, SciReader scans sentences to identify compound word phrases. When a word is selected, multiple definitions are returned with their database source and associated part of speech, if known. Importantly, for reading highlevel content, the definitions of words within the definition window can be identified by selecting the word. Since many definitions may still be too complicated for users with poor literacy, SciReader provides links to articles about a selected word from Wikipedia, Wiktionary, WebMD, MedScape, Google, and The Free Dictionary. Furthermore, a link to images for the word is also accessed through the application. These links provide additional depth should the definitions provided prove insufficient for comprehension. The application search bar can also be used as a medical or biological dictionary to retrieve the definition of individual words.

\section{Database Word Mapping Efficiency}

To determine the efficiency of word mapping in SciReader we loaded several scientific documents of similar length written for readers with varying levels of expertise. Results from analysis of a typical newspaper article, college level textbook, and biological journal article demonstrated that $98 \pm 1 \%(n=3)$ of all words are mapped with at least one definition; proper nouns were not included in this calculation (Table 1). To facilitate construction of a more comprehensive vocabulary, when a client selects a word for which there is no definition in the SciReader database, the word is recorded to a database table so that definitions can be added in the future.

Table 1 Evaluation of word mapping in SciReader

\begin{tabular}{|c|c|c|c|}
\hline Document & $\begin{array}{c}\# \\
\text { words }\end{array}$ & $\begin{array}{l}\text { \# word with } \\
\text { definitions }\end{array}$ & $\begin{array}{l}\text { Percent with } \\
\text { definitions }\end{array}$ \\
\hline $\begin{array}{l}\text { Judge Invalidates Human Gene Patent., J. Schwartz and A Pollack, The New York Times March 29, } \\
2010 .\end{array}$ & 772 & 768 & 100 \\
\hline $\begin{array}{l}\text { WormBook, The Online Review of C. elegans Biology., The C. elegans M. Chalfie and Research } \\
\text { Community, editors, Pasadena (CA): WormBook; 2005, Chapter 5.1. }\end{array}$ & 761 & 744 & 98 \\
\hline $\begin{array}{r}\text { Vyas J, Gryk MR, and Schiller. (2009). Venn, a tool for titrating sequence conservation onto protein } \\
\text { structures. Nucleic Acids Res. 37, e124. (results section) [23] }\end{array}$ & 800 & 781 & 98 \\
\hline Average & 778 & 764 & $98 \pm 1$ \\
\hline
\end{tabular}


Table 2 Survey responses to SciReader

\begin{tabular}{lcccccc}
\hline Question & Strongly Agree (1) & Agree (2) & Neutral (3) & Disagree (4) & Strongly Disagree (5) & Average Opinion \\
\hline Decreased reading time & $18.1 \%$ & $32.4 \%$ & $37.1 \%$ & $11.4 \%$ & $1.0 \%$ & 2.4 \\
\hline Increased comprehension & $23.8 \%$ & $52.4 \%$ & $21.9 \%$ & $1.0 \%$ & $1.0 \%$ & 2.0 \\
\hline
\end{tabular}

This table summarizes the responses of 105 individuals with a high school education.

The 5-point Likert scale is described in methods, scores are indicated in parentheses.

\section{Survey}

We surveyed 105 college students to determine if SciReader helped address problems in science/medical literacy (Table 2). Entry-level college students were chosen because these individuals have only been exposed to a high-school level of biology vocabulary. The students were provided access to seven chapters in an introductory level college biology text book and then asked to respond to whether SciReader helped reduce reading time or increased reading comprehension; two problems that are associated with poor scientific literacy [2]. Student selected their responses from a 5-point Likert scale indicating different levels of agreement with the statements provided (Table 2). Scores for the survey data are provided in Additional file 1. The opinion of SciReader users showed an average score of 2.4 with $50 \%$ of users indicating that the application reduced the time needed to read the chapters. A score of 2.0 was observed for increased reading comprehension where $76 \%$ of users thought that the application helped them better understand the chapter. These results indicate that SciReader is a tool that is perceived to be beneficial for reading technical content by the majority of users.

\section{Discussion}

Low medical and scientific literacy is a longstanding problem dating back to the late 1950s [12]. Most publications in these fields are focused upon identifying the problem [13-17], measuring literacy $[18,19]$, and assessing its impact on health care or education [20,21]. However, reports on progress toward improving literacy are generally limited. One example is the Medline Plus Kiosk, a community outreach project aimed at increasing medical literacy by presenting people with easy to comprehend medical information [22]. To further medical literacy we report the construction of SciReader, a new computational tool that can be used synergistically with internet applications. SciReader allows people to read medical content and obtain word definitions in the same view as the document being read.

SciReader is a unique tool that automates the tedious process of searching for, and evaluating scientific and medical terminology during the reading process. SciReader integrates a number of important text-based functions found in existing online dictionaries and ontologies, as well as search engines. A number of dictionaries and ontologies, which currently exist as separate sources are now accessible in a single search through the search engine embedded in SciReader. Typical content searches for images and detailed articles, normally performed with a search engine, are now coupled to selection of any word in SciReader. SciReader returns a series of related images from a Google search and also loads links to the Wikipedia encyclopedia and to articles from the WebMD and Medscape knowledgebases.

All of these functions can be accomplished without SciReader; however, integrating these tools into a unified view may have distinct advantages not realized in the separate applications by themselves. The recondite nature of scientific and medical content requires many readers to repetitively shift their train of thought and research the meanings of words. Not only is this a deterrent, but also negatively impacts, reading time, comprehension, and memory of the material read [5]. SciReader provides on the spot definitions and images for most words in a medical document. Even if the definition provided by SciReader does not help the reader, the search retrieves the images and links that a reader would normally pursue in the next attempts to ascertain comprehension.

One limitation in SciReader is that some of the definitions may be too complicated for a person with poor literacy to understand. In this situation, where more information is required, links to a WebMD, Wikipedia, or Wiktionary article and images about the topic are provided. Alternatively, a user can use Google. While these are not perfect solutions, they will facilitate learning more about the unknown word. Nevertheless, SciReader is a computational reading tool that can be used in conjunction with other web tools to promote medical/scientific literacy.

\section{Conclusions}

In summary, SciReader can be used to assist with interpreting medical documents for medical professionals and non-experts such as medical students, patients, and the general public. The application has the potential to improve health care by increasing their comprehension of medical and/or scientific literature so that patients can better understand their ailments and treatments.

\section{Availability and requirements}

- Project name: SciReader

- Project home page: http://scireader.bio-toolkit. com

- Operating system: Platform independent 
- Programming Language: Java, Flex

- Other requirements: Flash plug-in

- License: Free for academic use

- Any restriction to use by non-academics: License required

\section{Additional material}

Additional File 1: excel, SciReader Survey Data, This excel file contains the 5-point Likert scale data for the students surveyed.

\section{Acknowledgements and Funding}

This research was supported by National Institutes of Health grant GM079689. We thank David Sargeant for help administering the SciReader web site.

\section{Author details}

${ }^{1}$ School of Life Sciences, University of Nevada, Las Vegas, 4505 Maryland Pkwy., Las Vegas, NV 89154-4004 USA. ²Department of Molecular, Microbial, and Structural Biology, University of Connecticut Heath Center, 263 Farmington Ave., Farmington, CT 06030-3305 USA.

\section{Authors' contributions}

The application was designed and built by PG. Analysis was by PG and MS. MS prepared the manuscript. JV, RT, PG built the database. ML conducted the survey. MS conceptualized the study. All authors have read and approved the final manuscript.

\section{Competing interests}

The Board of Regents of the Nevada System of Higher Education, on behalf of the University of Nevada, Las Vegas, has filed a patent application that is pending. None of the authors have received compensation in any form that would be considered a competing interest nor is there any current plan to develop SciReader into a business. SciReader has not been licensed to any commercial company or government entity.

Received: 17 August 2010 Accepted: 25 January 2011

Published: 25 January 2011

\section{References}

1. Andrus MR, Roth MT: Health literacy: a review. Pharmacotherapy 2002, 22:282-302.

2. Gross L: Scientific illiteracy and the partisan takeover of biology. PLoS Biol 2006, 4:e167.

3. Scientific Literacy: How Do Americans Stack Up? [http://www.sciencedaily. com/releases/2007/02/070218134322.htm].

4. Human Development Report 2009. Palgrave Macmillian. New York; 2009

5. Knight S: Dictionary use while reading: the effects on comprehension and vocabulary acquisition for students of different verbal abilities. Mod. Lang. J 1994, 285-289.

6. Sigman M, Cecchi GA: Global organization of the Wordnet lexicon. Proc. Natl. Acad. Sci. USA 2002, 99:1742-1747.

7. Smith B, Ashburner M, Rosse C, Bard J, Bug W, Ceusters W, Goldberg L, Eilbeck K, Ireland A, Mungall C, Leontis N, Rocca-Serra P, Ruttenberg A, Sansone S, Scheuermann R, Shah N, Whetzel P, Lewis S: The OBO Foundry: coordinated evolution of ontologies to support biomedical data integration. Nature Biotech 2007, 25:1251-1255.

8. Fragoso G, de Coronado S, Haber M, Hartel F, Wright L: Overview and utilization of the NCl thesaurus. Comp. Funct. Genomics 2004, 5:648-654.

9. Lipscomb CE: Medical Subject Headings (MeSH). Bull Med Libr Assoc 2000, 88:265-266.

10. Ashburner M, Ball C, Blake J, Botstein D, Butler H, Cherry J, Davis A, Dolinski K, Dwight S, Eppig J, Harris M, Hill D, Issel-Tarver L, Kasarskis A, Lewis S, Matese J, Richardson J, Ringwald M, Rubin G, Sherlock G: Gene Ontology: tool for the unification of biology. Nat.Genet 2000, 25:25-29.
11. Sayers EW, Barrett T, Benson DA, Bolton E, Bryant SH, Canese K, Chetvernin V, Church DM, Dicuccio M, Federhen S, Feolo M, Geer LY, Helmberg W, Kapustin Y, Landsman D, Lipman DJ, Lu Z, Madden TL, Madej T, Maglott DR, Marchler-Bauer A, Miller V, Mizrachi I, Ostell J, Panchenko A, Pruitt KD, Schuler GD, Sequeira E, Sherry ST, Shumway M, Sirotkin K, Slotta D, Souvorov A, Starchenko G, Tatusova TA, Wagner L, Wang $Y$, John Wilbur W, Yaschenko E, Ye J: Database resources of the National Center for Biotechnology Information. Nucleic Acids Res 2010, 38 : D5-16.

12. Laugksch R: Scientific Literacy: A Conceptual Overview. Science Education 1999, 84:71-94.

13. Culliton BJ: The Dismal State of Scientific Literacy: Studies find only $6 \%$ of Americans and $7 \%$ of British meet standard for science literacy. Science 1989, 243:600.

14. Snow CE: Academic language and the challenge of reading for learning about science. Science 2010, 328:450-452.

15. Webb P: Science education and literacy: imperatives for the developed and developing world. Science 2010, 328:448-450.

16. Pearson PD, Moje E, Greenleaf C: Literacy and science: each in the service of the other. Science 2010, 328:459-463.

17. Zarcadoolas C, Pleasant A, Greer DS: Understanding health literacy: an expanded model. Health Promot Int 2005, 20:195-203.

18. Ashida S, Goodman M, Pandya C, Koehly LM, Lachance C, Stafford J, Kaphingst KA: Age Differences in Genetic Knowledge, Health Literacy and Causal Beliefs for Health Conditions. Public Health Genomics 2010.

19. McCormack L, Bann C, Squiers L, Berkman ND, Squire C, Schillinger D, Ohene-Frempong J, Hibbard J: Measuring health literacy: a pilot study of a new skills-based instrument. J Health Commun 2010, 15(Suppl 2):51-71.

20. Garcia SF, Hahn EA, Jacobs EA: Addressing low literacy and health literacy in clinical oncology practice. J Support Oncol 2010, 8:64-69.

21. Paasche-Orlow MK, Wolf MS: Promoting health literacy research to reduce health disparities. J Health Commun 2010, 15(Suppl 2):34-41.

22. Teolis MG: A MedlinePlus Kiosk Promoting Health Literacy. J Consum Health Internet 2010, 14:126-137.

23. Vyas J, Gryk MR, Schiller MR: VENN, a tool for titrating sequence conservation onto protein structures. Nucleic Acids Res 2009, 37:e124

\section{Pre-publication history}

The pre-publication history for this paper can be accessed here: http://www.biomedcentral.com/1472-6947/11/4/prepub

doi:10.1186/1472-6947-11-4

Cite this article as: Gradie et al: SciReader enables reading of medical content with instantaneous definitions. BMC Medical Informatics and Decision Making 2011 11:4.

\section{Submit your next manuscript to BioMed Central and take full advantage of:}

- Convenient online submission

- Thorough peer review

- No space constraints or color figure charges

- Immediate publication on acceptance

- Inclusion in PubMed, CAS, Scopus and Google Scholar

- Research which is freely available for redistribution 